REF - ISSN 1808-0804 Vol. V (3), 46 - 53, 2008

\title{
AUSÊNCIA DE EFEITO TÓXICO-GENÉTICO DE Morinda citrifolia (NONI) EM CÉLULAS SOMÁTICAS DE Drosophila melanogaster.
}

\author{
Absence of genotoxicity effect of Morinda citrifolia (Noni) in \\ somatic cells of Drosophila melanogaster.
}

\author{
Leonardo P. Franchi ${ }^{1}$; Nilza N. Guimarães ${ }^{1}$; Maurício Lehmann²; \\ Heloísa Helena R. de Andrade ${ }^{2}$; Kênya S. Cunha ${ }^{1 \star}$. \\ ${ }^{1}$ Laboratório de Genética Toxicológica, Departamento de Bioquímica e Biologia Molecular, ICB II, \\ Universidade Federal de Goiás (UFG), Goiânia-GO. ${ }^{2}$ Laboratório da Toxicidade Genética (TOXIGEN), \\ Universidade Luterana do Brasil (ULBRA).
}

*Autor para correspondência: e-mail: kenya@icb.ufg.br

Recebido em 05/06/2008 - Aceito em 01/12/2008

RESUMO: Na medicina popular, o suco produzido a partir do fruto de Morinda citrifolia (Noni) é conhecido por prevenir e curar várias doenças tais como diabetes, diarréia, dores, hipertensão, artrite, estresse e câncer. Mais de 160 constituintes químicos já foram identificados no Noni, entre eles estão asperulosido, escopoletina e antraquinonas, além de vitaminas e vários aminoácidos. Devido à ausência de informações no que se refere à possível indução de alterações no genoma celular em nível de mutação e recombinação mitótica, realizou-se uma avaliação tóxico-genética do suco de Noni. Assim, foi empregado o Teste para Detecção de Mutação e Recombinação (SMART) em células somáticas de Drosophila melanogaster, que possibilita a avaliação, simultânea e in vivo, de efeitos mutagênicos e recombinogênicos. Utilizando o cruzamento padrão (ST), foram obtidas larvas de terceiro estágio que receberam tratamento crônico, via alimentação. As larvas foram tratadas com quatro diferentes concentrações do suco de Noni (25, 50, 75 e 100\% v/v); água destilada (controle negativo) e mitomicina $C$ (controle positivo). Após a eclosão das pupas, foram analisados os tricomas presentes nas asas dos indivíduos adultos, visando a identificação e quantificação de alterações fenotípicas. Foram realizados dois experimentos independentes utilizando duas repetições por concentração testada. Os resultados de cada experimento foram testados estatisticamente pelo teste binomial condicional, no qual os dados dos diferentes tratamentos foram comparados com o controle negativo. Os resultados dos dois experimentos indepedentes foram somados, considerando-se que a aplicação do teste de Kruskal-Wallis demonstrou a ausência de diferenças estatísticas entre os dois experimentos. Um total de 301 indivíduos foram analisados e os resultados obtidos demonstraram que o suco de Noni não apresentou efeito tóxico-genético em células somáticas de Drosophila melanogaster.

PALAVRAS-CHAVE: Mutagênese, Drosophila melanogaster, Morinda citrifolia, Noni

\begin{abstract}
In the popular medicine, the juice produced from the fruit of Morinda citrifolia (Noni) is known to prevent and cure many diseases such as diabetes, diarrhea, pain, hypertension, arthritis, stress and cancer. More than 160 chemical constituents have been identified in Noni, including asperuloside, escopoletin and anthraquinones, and several vitamins and amino acids. Due to the lack of information about the possible induction of alterations in the cellular genome in the level of mutation and mitotic recombination, the genotoxicity of Noni juice was evaluated. Thus, it was applied the Somatic Mutation And Recombination Test (SMART) in somatic cells of Drosophila melanogaster, which allows to evaluate, simultaneously and in vivo, the mutagenic and recombinogenic effects. Using the standard cross (ST), it was obtained third-instar larvae that were treated chronically by feeding. The larvae were treated with four different concentrations of Noni juice $(25,50,75$ and $100 \% \mathrm{v} / \mathrm{v}$ ), distilled water (negative control) and mitomycin C (positive control). After pupa eclosion, the wings trichomes of adults were analyzed, aiming the identification and quantification of phenotypic alterations. Two independent experiments were carried using two repetitions per tested concentration. The results of each experiment were statistically tested with binomial conditional test in which the different treatments were compared with the negative control. The results of both independent experiments were polled, considering that the KruskalWallis test demonstrated the absence of statistic differences between both experiments. In a total of 301 individual
\end{abstract}


Franchi, L. P. et al./Revista Eletrônica de Farmácia Vol 5(3), 46 - 53, 2008.

were analysed and the results demonstrated that the Noni juice has no genetic toxicity effects in somatic cells of Drosophila melanogaster.

KEYWORDS: Mutagenesis, Drosophila melanogaster, Morinda citrifolia, Noni

\section{INTRODUÇÃO}

Existem numerosos compostos presentes em nosso meio ambiente, que são potencialmente mutagênicos e que podem interagir entre si e, assim, atuarem sobre o DNA das células vivas. A estreita correlação existente entre mutagenicidade e carcinogenicidade ressalta a atividade mutagênica de um determinado agente, químico ou físico, não somente como um risco para as gerações futuras, mas também como um fator que induz a formação de tumores malignos nos organismos expostos às substâncias mutagênicas (WÜRGLER et al., 1984). $\mathrm{Na}$ verdade, a maioria dos carcinógenos inicia sua atividade tumoral através de interações específicas com o DNA das células - gerando lesões genéticas permanentes que, geralmente, se expressam como mutações gênicas e/ou aberrações cromossômicas (BARTSCH et al., 1980).

Uma vez que a incidência de doenças genéticas e câncer podem ser reduzidos pelo decréscimo nas taxas de mutação, uma das estratégias para proteção das populações humanas é a identificação de agentes mutagênicos e/ou carcinogênicos, seguida da implementação de medidas de controle do risco genético - através da diminuição da exposição humana a estes agentes (WATERS et al., 1990).

A utilização de plantas como recurso terapêutico é uma prática generalizada na medicina popular e tem aumentado acentuadamente nas últimas décadas. Apesar da ampla utilização das plantas medicinais, pouca informação se encontra disponível sobre seus constituintes (BATISTA et al., 2005; FABRICANT \& FARNSWORTH, 2001; MACIEL et al., 2002; PRADO et al., 2005), bem como sobre os riscos em potencial oferecidos à saúde humana (OLIVEIRA \& GONÇALVES, 2006). Além disso, nos últimos anos, tem sido demonstrado que uma série de substâncias, tais como antraquinonas, taninos e flavonóides (BROWN, 1980), encontradas em plantas utilizadas na medicina popular, como por exemplo, a jaboticaba-do-cerrado, é capaz de: (i) induzir a ocorrência de eventos mutacionais em tecidos somáticos ou germinais (PEREIRA et al., 2008; FAGUNDES et al., 2005; SANTOS et al., 2008; VERSCHAEVE \& VAN STADEN; 2008), podendo levar ao desenvolvimento de doenças somáticas, efeitos teratogênicos e a desordens hereditárias múltiplas; ou (ii) modular os efeitos mutagênicos e/ou carcinogênicos de uma série de agentes genotóxicos (KNASMÜLLER et al., 2002, POERSCH et al., 2007; ROSA et al., 2007).

Morinda citrifolia, comumente conhecida como Noni, tem uma longa história de utilização medicinal na Polinésia, China, Índia, Austrália e Havaí. E recentemente, tornou-se um suplemento alimentar, em forma de suco, bastante consumido nos Estados Unidos, Japão e parte da Europa. O crescimento em popularidade pode ser em parte atribuído ao seu poder "cura-tudo". Este suco vem sendo utilizado na medicina popular no tratamento de doenças e/ou distúrbios tais como: diabetes, diarréia, dores, hipertensão, artrite, estresse e câncer (SU et al., 2005).

Cerca de 160 fitoconstituintes já foram identificados na planta Morinda citrifolia, e os principais são compostos fenólicos, ácidos orgânicos e alcalóides. Dos compostos fenólicos, os mais importantes relatados são as antraquinonas (damnacanthal, morindona, morindina) e também asperulosido e escopoletina (WANG \& SU, 2001).

CHAN-BLANCO et al. (2006) relataram que em testes in vivo e in vitro o suco de Noni apresentou atividades anti-microbiana, anti-câncer, anti-oxidante, anti-inflamatória, analgésica e cardiovascular. Saludes et al. (2002) descreveu que o extrato etanólico do Noni possui efeito contra a tuberculose sendo capaz de inibir de 89-95\% do crescimento de Mycobacterium tuberculosis. HIRAZUMI et al. (1996), e HIRAZUMI \& FURUSAWA (1999), utilizando um precipitado de etanol do suco de Noni, encontraram efeitos imunomodulatório e anti-tumor contra o carcinoma de pulmão de Lewis.

Entretanto, STADLBAUER et al. (2005) relataram dois casos de hepatotoxicidade do suco de Noni. Um homem de 29 anos de idade com hepatite tóxica prévia associada a pequenas doses de paracetamol desenvolveu insuficiência hepática após o consumo de 1,5 L de suco de Noni ao longo de 3 semanas, e necessitou de transplante de fígado com urgência. E, uma mulher de 62 anos de idade sem evidências de doenças hepáticas prévias desenvolveu um episódio de hepatite aguda após consumo de $2 \mathrm{~L}$ de suco de Noni distribuídos no período de abril a julho de 2003. YÜCE et al. (2006) e MILLONIG et al. (2005) relataram mais dois casos de hepatotoxicidade após o consumo do suco de Noni. PAWLUS et al. (2005) e STADLBAUER et al. (2005) sugerem que as antraquinonas são os componentes mais prováveis pela hepatotoxicidade induzida por extratos de Morinda citrifolia. Sabe-se que Morinda citrifolia contém em suas raízes antraquinonas genotóxicas. No entanto, o suco da fruta do Noni não apresentou efeitos mutagênicos em fibroblastos de hamster Chinês (WESTENDORF et al., 2005). E também não aumentou significativamente a freqüência de aberrações cromossômicas em linfócitos humanos (EDWARDS, 2003).

Portanto, diante da vasta utilização do suco de Noni pela população, em diversas regiões do mundo, e devido à pequena quantidade de informações no que se refere à possível indução de alterações no genoma 
Franchi, L. P. et al./Revista Eletrônica de Farmácia Vol 5(3), 46 - 53, 2008.

celular em nível de mutação gênica, cromossômica e recombinação mitótica, torna-se necessário uma avaliação genotóxica do produto.

\section{MATERIAIS E MÉTODOS}

Foi utilizado nesse trabalho o suco de Noni (TAHITIAN NONI ${ }^{\circledR}$ Juice) fabricado por Morinda, Inc e introduzido no mercado mundial em 1996. O preparo das soluções foi realizado à hora do tratamento, diluindo-se o suco de Noni em água destilada em três diferentes concentrações $(25,50,75$ e 100\% v/v). A água destilada foi utilizada como controle negativo, e mitoci ${ }^{\circledR}$ (Bristol - mitomicina C - MMC - CAS N. 50-07-7), na concentração de $0,05 \mathrm{mM}$, dissolvida em água destilada, foi utilizada como controle positivo para demonstrar a eficiência de indução de genotoxicidade nos experimentos.

\section{Avaliação do potencial genotóxico do suco de Noni}

Primeiramente foi realizado um teste de sobrevivência, no qual 100 larvas por tubo de tratamento foram expostas às diferentes concentrações de suco de Noni. $O$ número de moscas sobreviventes por tratamento fornece uma indicação da toxicidade do composto.

Para avaliar a atividade mutagênica e/ou recombinogênica do Noni utilizou-se o Teste para Detecção de Mutação e Recombinação (SMART) em células somáticas de Drosophila melanogaster. O teste SMART de asa de Drosophila melanogaster foi desenvolvido por GRAF et al. (1984), sendo considerado um teste rápido, barato, e que produz resultados confiáveis e altamente reproduzíveis.

A D. melanogaster apresenta as vantagens de ter um curto período de geração, o que possibilita a rapidez do teste, um pequeno número de cromossomos, linhagens geneticamente bem caracterizadas, e um sistema enzimático semelhante à fração S9 encontrada em mamíferos, que permite o metabolismo de agentes xenobióticos (CLARK 1982; GRAF et al., 1984; HÄLLSTRÖM et al., 1984). Dentre os citocromos P450 (CYP) destaca-se a família CYP6 de Drosophila melanogaster, que possui regiões altamente similares com citocromos pertencentes à família CYP3 que, juntamente com CYP2, são responsáveis pelo metabolismo de drogas em vertebrados (DANIELSON et al. 1997; 1998).

Este teste detecta a perda da heterozigose, que pode ocorrer espontaneamente ou ser induzida por agentes físicos e químicos, em células primordiais dos discos imaginais de asas, no período de larva. No SMART de asa podem ser utilizadas as linhagens de Drosophila melanogaster mwh e $\mathrm{flr}^{3}$. A linhagem mwh, com constituição genotípica $m w h / m w h$ possui o gene marcador $m w h$ que se localiza no cromossomo $3(3-3,0)$ e que tem sua manifestação fenotípica caracterizada por três ou mais pêlos por célula da asa. A linhagem $\mathrm{flr}^{3} \mathrm{com}$ constituição genotípica $f r^{3} / I n(3 L R) T M 3$, ri $p^{p} \operatorname{sep~I(3)89Aa~} b x^{34 e}$ e $B d^{S}$, possui o gene marcador $f / r^{3}$ localizado no braço esquerdo do cromossomo 3 , em uma região mais proximal (3-38,8). A expressão fenotípica do gene $\mathrm{flr}^{3}$ afeta a forma dos pêlos dando-lhes uma aparência de "chama de vela". Além disso, a linhagem $\mathrm{flr}^{3}$ possui o gene marcador $B d^{S}$, que confere um formato serrilhado às asas, e um cromossomo balanceador denominado TM3 que contém múltiplas inversões (ANDRADE et al., 2003).

Os experimentos utilizaram o cruzamento padrão (ST), o qual foi realizado através de cruzamentos entre fêmeas virgens da linhagem $\mathrm{flr}^{3}$ e machos $\mathrm{mwh}$. Neste caso, analisou-se o efeito de genotoxinas de ação direta justificado pela presença, nessas linhagens, de níveis basais de enzimas de metabolização do tipo CYP6A2 (SANER et al., 1996), que corresponde à subfamília CYP3A em humanos (AOYAMA et al., 1989) e CYP3A16 em camundongos (ITOH et al., 1994). Tal cruzamento produziu dois tipos de progênie: (i) indivíduos transheterozigotos para os genes marcadores $(\mathrm{MH})$, com constituição genotípica $m w h+/+f / r^{3}$; e (ii) indivíduos heterozigotos para o cromossomo TM3 $(\mathrm{BH})$, constituídos por $m w h+1+\mathrm{TM} 3, B d^{s}$. Devido à presença do cromossomo balanceador TM3, as células resultantes de recombinação mitótica são inviáveis nestes indivíduos (GRAF et al., 1984; ANDRADE et al., 2003). Os indivíduos BH diferenciam-se fenotipicamente dos indivíduos MH pela presença de recortes na borda das asas, característica conferida pelo marcador $B d^{S}$, deixando-as com aspecto serrilhado (GRAF et al., 1984).

Assim, a análise dos indivíduos MH permite detectar a ocorrência de mutações gênicas, pequenas mutações cromossômicas e recombinações mitóticas proximais e distais. A análise dos indivíduos BH permite detectar somente a ocorrência de mutações gênicas e pequenas mutações cromossômicas. Através da comparação dos resultados obtidos nos indivíduos $\mathrm{MH}$ com os resultados obtidos nos indivíduos $\mathrm{BH}$ pode-se quantificar a real contribuição da recombinação para a genotoxicidade total do composto em estudo. Neste estudo foi analisado um total de 301 indivíduos $\mathrm{MH}$.

\section{Tratamento Crônico}

Os cruzamentos das linhagens foram realizados em massa (80 fêmeas x 40 machos), durante 3 dias, em vidros contendo meio de cultura padrão. Após este período, os casais foram transferidos para frascos contendo meio de ovoposição, onde permaneceram por 8 horas, sendo em seguida descartados. Passadas $72 \pm 4$ horas do início do período de ovoposição foram coletadas as larvas de terceiro estágio, por flotação em água corrente. 
Estas larvas foram, então, colocadas em frascos de tratamento contendo $0,9 \mathrm{~g}$ de meio sintético onde foram acrescentados $3 \mathrm{~mL}$ das soluções de tratamento. As larvas permaneceram em tratamento por aproximadamente 48 horas - o que caracteriza o tratamento crônico do ensaio - até atingirem o estágio de pupa. Através deste procedimento experimental, as células dos discos imaginais, que originaram as asas dos adultos, ficaram expostas às diferentes soluções por 5 a 6 ciclos de divisão mitótica - o que corresponde a $95 \%$ de todas as divisões celulares, que ocorrem desde o desenvolvimento do embrião até o início de pupação (FREl et al., 1992). Os adultos que eclodiram das pupas 6 a 7 dias depois do tratamento, foram contados e conservados em álcool $70 \%$, até a montagem das lâminas.

\section{Análise microscópica e avaliação tóxico-genética}

As lâminas das asas dos adultos tratados foram montadas em solução de Faure [goma arábica (30 $\mathrm{g})$, glicerol $(20 \mathrm{~mL})$, água $(50 \mathrm{~mL})$ e hidrato de cloral $(50 \mathrm{~g})$ ] e analisadas em microscópio óptico com aumento de 400 vezes (GRAF et al., 1984). A análise dos tricomas, presente nas superfícies dorsal e ventral das asas, permitiu a identificação de manchas de pêlos mutantes (Figura 1) que podem ser classificadas como:

(i) simples (pequenas - com 1-2 células mutantes - ou grandes - com 3 ou mais células mutantes): expressando o fenótipo mutante $m w h$ ou $f r^{3}$ - indicando a ocorrência de mutações gênicas, alterações cromossômicas e recombinação mitótica;

(ii) gêmeas: formadas por células adjacentes $m w h$ e $f l r^{3}$ - originadas exclusivamente por recombinação, o que significa que este tipo de mancha pode fornecer indicações da ação recombinogênica do composto.

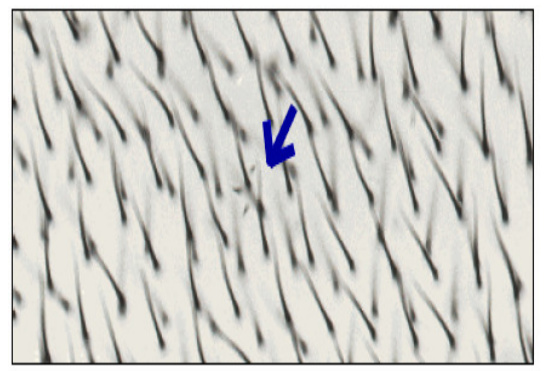

Mancha simples pequena

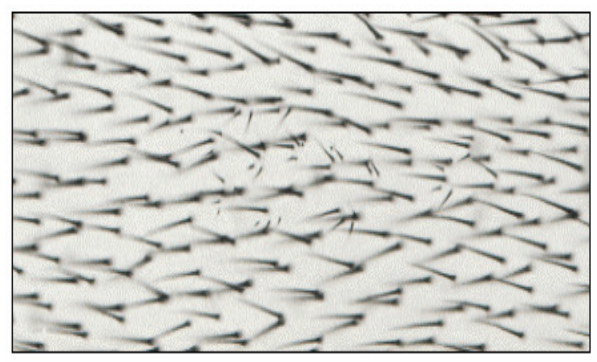

Mancha simples grande

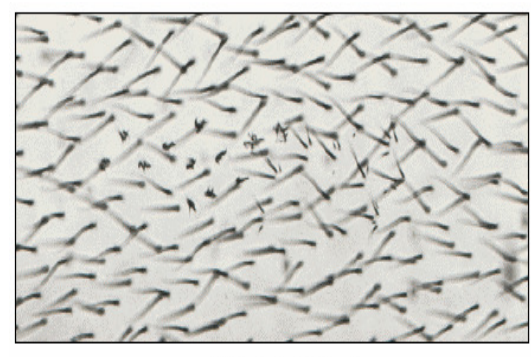

Mancha gêmea

Figura 1. Classificação das manchas de acordo com o fenótipo e o número de células contendo de pêlos mutantes.

\section{Análise estatística}

Para avaliar a significância estatística dos resultados obtidos, foi realizado o procedimento proposto por FREI \& WÜRGLER (1988), uma análise de múltiplas decisões que gera quatro diferentes diagnósticos: positivo, fraco positivo, negativo ou inconclusivo (nível de significância $p<0,05$ ). A freqüência de cada tipo de mancha mutante por indivíduo de uma série tratada foi comparada com seu respectivo controle negativo, usando o teste Binomial Condicional de KASTEMBAUM \& BOWMAN (1970). Para avaliar resultados negativos, fatores de multiplicação $(m)$ foram introduzidos no teste. São eles: $m=2$ para manchas simples pequenas e total de manchas, devido às suas altas freqüências espontâneas e $m=5$ para manchas simples grandes e gêmeas, que raramente surgem de forma espontânea (FREI \& WÜRGLER 1988; FREI et al., 1992; GRAF et al., 1984). Desta forma são estabelecidos os critérios para o diagnóstico positivo, que requer uma freqüência de mutações no tratado que seja $m$ vezes maior que a freqüência obtida no controle negativo (ANDRADE et al., 2003).

\section{RESULTADOS E DISCUSSÃo}

Após a exposição ao suco de Noni as larvas dos tubos de tratamento foram contadas e o número de indivíduos sobreviventes foi de $100 \%$ em todas as concentrações utilizadas (resultados não apresentados) demonstrando a ausência de toxicidade do Noni nas doses testadas.

Os resultados obtidos nos experimentos de avaliação tóxico-genética com diferentes concentrações do suco de Noni, MMC $(0,05 \mathrm{mM})$ e água destilada estão apresentados na Tabela 1. As freqüências de manchas espontâneas (água destilada) e do controle positivo (MMC) alcançaram valores que são compatíveis com os resultados apresentados na literatura (GRAF et al., 1984; SANTOS et al., 1999). Estes resultados demonstram que o sistema responde adequadamente aos parâmetros necessários para que a análise mutagênica seja realizada. 
Franchi, L. P. et al./Revista Eletrônica de Farmácia Vol 5(3), 46 - 53, 2008.

Para a interpretação dos resultados utilizou-se a freqüência do total de manchas (TM). Sendo assim, o TM observados nos indivíduos MH tratados com diferentes concentrações de Noni $(25,50,75$ e $100 \% \mathrm{v} / \mathrm{v})$ não foram estatisticamente significativas $(P>0,05)$, quando comparadas com a freqüência de manchas observadas no controle negativo (Tabela 1). Desta forma não se justifica a análise dos indivíduos $\mathrm{BH}$.

Considerando os resultados negativos no TM analisadas nos tratamentos, podemos concluir que o suco de Noni foi incapaz de induzir toxicidade genética relacionadas com mutações gênicas, cromossômicas e/ou eventos recombinacionais em células somáticas de Drosophila melanogaster. Estes resultados corroboram com os resultados encontrados por EDWARDS (2003) \& WESTENDORF et al. (2005), que detectaram a ausência de mutagenicidade em fibroblastos de hamster Chinês (V79/HPRT) e em linfócitos humanos, respectivamente. TABELA 1: Avaliação dos efeitos mutagênico e/ou recombinogênico do suco de Noni em
células somáticas de larvas MH provenientes do cruzamento ST.

Genótipo Manchas por indivíduo ( $\mathrm{n}^{\circ}$ de manchas) diagnóstico estatístico ${ }^{\mathrm{a}}$

\begin{tabular}{|c|c|c|c|c|c|c|c|c|c|c|c|c|c|}
\hline \multirow{2}{*}{$\begin{array}{c}\text { Concentrações } \\
m w h / f l r^{3}\end{array}$} & \multirow[t]{2}{*}{$\begin{array}{c}\text { Moscas } \\
(\mathbf{N})\end{array}$} & \multicolumn{2}{|c|}{$\begin{array}{c}\text { MSP } \\
\text { (1-2 céls) } \\
m=2\end{array}$} & \multicolumn{3}{|c|}{$\begin{array}{c}\text { MSG } \\
\text { (>2 céls) } \\
m=5\end{array}$} & \multicolumn{3}{|c|}{$\begin{array}{c}\text { MG } \\
m=5\end{array}$} & \multicolumn{3}{|c|}{$\begin{array}{c}\text { TM } \\
m=2\end{array}$} & \\
\hline & & & & & & & & & & & & & \\
\hline $\begin{array}{l}\text { Contr. Negativo } \\
\qquad\left(\mathrm{H}_{2} \mathrm{O}\right)\end{array}$ & 53 & 0,72 & (38) & & 0,13 & $(07)$ & & 0,02 & $(01)$ & & 0,87 & (46) & \\
\hline $\begin{array}{l}\text { Contr. Positivo } \\
\text { (MMC } 0,05 \mathrm{mM})\end{array}$ & 36 & 12,28 & $(442)$ & + & 7,72 & $(278)$ & + & 4,08 & (147) & + & 24,08 & (867) & + \\
\hline Noni $25 \%$ (v/v) & 53 & 0,87 & (46) & - & 0,04 & $(02)$ & - & 0,00 & $(00)$ & $\mathrm{i}$ & 0,91 & (48) & - \\
\hline Noni 50\% (v/v) & 53 & 0,51 & (27) & - & 0,17 & (09) & $\mathrm{i}$ & 0,02 & (01) & $\mathrm{i}$ & 0,70 & (37) & - \\
\hline Noni 75\% (v/v) & 53 & 0,57 & (30) & - & 0,09 & $(05)$ & $\mathrm{i}$ & 0,02 & $(01)$ & $\mathrm{i}$ & 0,68 & (36) & - \\
\hline Noni $100 \%(\mathrm{v} / \mathrm{v})$ & 53 & 0,70 & (37) & - & 0,15 & $(08)$ & $\mathrm{i}$ & 0,04 & $(02)$ & $\mathrm{i}$ & 0,89 & (47) & - \\
\hline
\end{tabular}

\section{CONCLUSÕES}

A estratégia experimental utilizada neste trabalho permitiu evidenciar a ausência de efeitos tóxicogenéticos que estão intimamente relacionados com a indução de diversas doenças somáticas, dentre elas as neoplasias. Considerando-se alguns relatos da literatura que apontam efeitos benéficos do Noni (CHANBLANCO et al., 2006; HIRAZUMI \& FURUSAWA, 1999), este trabalho abre uma nova perspectiva de avaliação relativa aos possíveis efeitos anti-genotóxicos induzidos pelo Noni.

\section{REFERÊNCIAS BIBLIOGRÁFICAS}

ANDRADE, H. H. R.; LEHMANN, M. Teste para detecção de mutação e recombinação somática (SMART) em Drosophila melanogaster. In RIBEIRO, L. R., SALVADORI, D. M. F., MARQUES, E. K., Mutagênese ambiental, Canoas: Editora ULBRA, Edição Única, p.281-307, 2003.

AOYAMA, T.; YAMANO, S.; WAXMAN, D.J.; LAPENSON, D.P.; MEYER, V.A.; FISCHER, V.; TYNDALE, R.; INABA, T.; KALOW, W.; GELBION, H.V.; GONZALEZ, F.J. Cytochrome P450 hPCN3, a novel cytochrome P450 IIIA gene product that is differentially expressed in adult human liver. Journal of Biological Chemistry, v.264, p.10388-10395.

BARTSCH, H.; MALAVEILLE, C.; CAMUS, A. M.; MARTEL-PLANCHE, G.; BRUN, G.; HAUTEFEILLE, A.; SABADIE, N.; BARBIN, A.; DREVON, T.; PICOLLO, C.; MONTESANO, R. Validation and comparative studies on 180 chemicals with $S$. typhimurium strains and V-79 Chinese hamster cells in the presence of various metabolizing systems. Mutation Research, v.76, p. 1-50, 1980. 
Franchi, L. P. et al./Revista Eletrônica de Farmácia Vol 5(3), 46 - 53, 2008.

BATISTA, A. C. M.; RIBEIRO, P. A. M.; ARANTES, M. C. B.; OLIVEIRA, L. M. G.; FREITAS, M. R. F.; SILVA, L. N. M.; NOGUEIRA, J. C. M.; PAULA, J. R.; BARA, M. T. F. Estudo farmacognóstico de Tinospora cordifolia (wild) Miers ex Hook. F. \& Thoms, Menispermaceae. Revista Eletrônica de Farmácia, v. 2 (2), p. 34-36, 2005.

BROWN, J. P. A review of the genetic effects of naturally occurring flavonoids, anthraquinones and related compounds. Mutation Research, v. 75, p. 243-277, 1980.

CHAN-BLANCO, Y.; VALIANT, F.; PEREZ, M. A.; REYNES, M.; BRILLOUET.; BRAT, P. The noni fruit (Morinda cintrifolia L.): A review of agricultural research, nutritional and therapeutic properties. Journal of food compositions and analysis, v. 19, p. 645-654, 2006.

CLARK, A. M. The use of larval stages of Drosophila in screening for some naturally occurring mutagens. Mutation Research, v. 92, p. 89-97, 1982.

DANIELSON, P. B.; FOSTER, J. L. M.; MCMAHILL, M. M.; SMITH, M. K.; FOGLEMAN, J. C. Induction by alkaloids and phenobarbital of Family 4 cytochrome P450s in Drosophila: evidence for involvement in host plant utilization. Molecular and General Genetics, v. 259, p. 54-59, 1998.

DANIELSON, P. B.; MACINTYRE, R. J.; FOGLEMAN, J. C. Molecular cloning of a family of xenobiotic-inducible drosophilid cytochrome P450s: evidence for involvement in host-plant allelochemical resistance. Proccedings of the National Academy of Science, v. 94, p. 10797-10802, 1997.

EDWARDS, C. N. In vitro mammalian chromosome aberration test performed with human lymphocytes. Test Report. Scantox Biologisk Laboratorium. Lille Skensved, DK (Lab no. 48877), 2003.

FABRICANT, D. S.; FARNSWORTH, N. R. The Value of Plants Used in Traditional Medicine for Drug Discovery. Environmental Health Perspectives v. 109, p. 69-75, 2001.

FAGUNDES, F. A.; OLIVEIRA, L. B.; CUNHA, L. C.; VALADARES, M. C. Annona coriacea induz efeito genotóxico em camundongos. Revista Eletrônica de Farmácia, v.2 (1), p. 24-29, 2005.

FARINE, J. P.; LEGAL, L.; MORETEAU, B.; LE QUERE, J. L. Volatile components of ripe fruits of Morinda citrifolia and their effects on Drosophila. Phytochemistry, v. 41, p. 433-8, 1996.

FREI, H., CLEMENTS, J., HOWE, D.; WÜRGLER, F. E. The genotoxicity of the anti-cancer drug mitoxantrone in somatic and germ cells of Drosophila melanogaster. Mutation Research/Genetic Toxicology, v. 279, p. 21-33, 1992.

FREI, H.; WURGLER, F. E. Statistical methods to decide whether mutagenicity test data from Drosophila assay indicate a positive, negative, or inconclusive result. Mutation Research, v. 203, p. 297-308, 1988.

GRAF, U.; WURGLER, F. E.; KATZ, A. J.; FREI, H.; HALL, C. B.; KALE, P. G. Somatic Mutation and Recombination Test in Drosophila melanogaster. Enviromental Mutagenesis, v. 6, p. 153-188, 1984.

HÄLLSTRÖM, I.; BLANCK, A.; ATUMA, S. Genetic variation in cytochrome P-450 and xenobiotic metabolism in Drosophila melanogaster. Biochemical Pharmacology, v. 33, p. 13-20, 1984.

HIRAZUMI, A. \& FURUSAWA, E. An immunomodulatory polysaccharide-rich substance from the fruit juice of Morinda citrifolia (Noni) with antitumor activity. Phytotherapic Reseacher, v. 13, p. 380-387, 1999.

HIRAZUMI, A.; FURUSAWA, E., CHOU, S.C, HOKAMA, Y. Immunomodulation contributes to the anticancer activity of Morinda citrifolia (Noni) fruit juice. Proceedings of the Western Pharmacological Society, v. 39, p. 7-9, 1996.

ITOH, S.; SATOH, M., ABE, Y., HASHIMOTO, H., YANAGOMITO T., KAMATAKI, T. A novel form of maouse cytochrome-P450 3A (cyp 3A-16).Its cDNA cloning and expression in fetal liver. European Journal of. Biochemistry, v. 226, p.877-882, 1994.

KASTENBAUM, M.A., BOWMAN, K.O. Tables for determining the statistical significance of mutation frequencies. Mutation Research, v. 9, p. 527-549, 1970. 
Franchi, L. P. et al./Revista Eletrônica de Farmácia Vol 5(3), 46 - 53, 2008.

KNASMÜLLER, S.; STEINKELLNER, H.; MAJER, B. J.; NOBIS, E. C.; SCHARF, G.; KASSIE, F. Search for dietary antimutagens and anticarcinogens: methodological aspects and extrapolation problems. Food and Chemical Toxicology, v. 40, p. 1051-1062, 2002.

MACIEL, M. A. M.; PINTO, A. C.; VEIGA JR., V. F. Plantas medicinais: a necessidade de estudos multidisciplinares. Química Nova, v. 25, p. 429-438, 2002.

MILLONIG, G.; STADLMANN, S.; VOGEL, W. Herbal hepatotoxicity: acute hepatitis caused by a Noni preparation (Morinda citrifolia). European Journal of Gastroenterology \& Hepatology. v. 17(4), p. 445-447, 2005.

OLIVEIRA, F. Q. \& GONÇALVES, L. A. Conhecimento sobre plantas medicinais e fitoterápicos e potencial de toxicidade por usuários de Belo Horizonte, Minas Gerais. Revista Eletrônica de Farmácia, v. 3 (2), p. 36-41, 2006.

PAWLUS, A. D.; SU, B. N.; KELLER, W. J.; KINGHORN, A. D. An anthraquinone with potent quinone reductaseinducing activity and other constituents of the fruits of Morinda citrifolia (noni). Journal of Natural Products, 68 (12), 1720-1722, 2005.

PEERZADA, N., RENAUD, S., RYAN, P. Vitamin C and elemental composition of some bushfruits. Journal of Plant Nutrition, v. 13 p. 787-93, 1990.

PEREIRA, A. D.; ANDRADE, S. F.; SWERTS, M. S. O.; MAISTRO, E. L. First in vivo evaluation of the mutagenic effect of Brazilian green propolis by comet assay and micronucleus test. Food and Chemical Toxicology, v. 46, p. 2580-2584, 2008.

POERSCH, A.; SANTOS, F. V.; MACIEL, M. A. M.; CÂMARA, J. K. P.; DANTAS, T. N. C.; CÓlUS, I. M. S. Protective effect of DCTN (trans-dehydrocrotonin) against induction of micronuclei and apoptosis by different mutagenic agents in vitro Mutation Research/Genetic Toxicology and Environmental Mutagenesis, v. 629, p.1423, 2007.

PRADO, C. C.; ALENCAR, R. G.; PAULA, J. R.; BARA, M. T. F. Avaliação do teor de polifenóis da Camellia sinensis (chá verde). Revista Eletrônica de Farmácia, v. 2 (2), p. 164-167, 2005.

ROSA, R. M.; MOURA, D. J.; MELECCHI, M. I. S.; SANTOS, R. S.; RICHTER, M. F.; CAMARÃO, E. B.; HENRIQUES, J. A. P.; RAMOS, A. L. L. P.; SAFFI J. Protective effects of Hibiscus tiliaceus L. methanolic extract to V79 cells against cytotoxicity and genotoxicity induced by hydrogen peroxide and tert-butyl-hydroperoxide, Toxicology in Vitro, v. 21, p. 1442-1452, 2007.

SALUDES, J.P.; GARSON, M.J.; FRANZBLAU, S.G.; AGUINALDO, A.M. Antitubercular constituents from the hexane fraction of Morinda citrifolia L. (Rubiaceae). Phytotherapic Research, v. 16, p. 683-685, 2002.

SANER, C.; WEIBEL, B.; WÜRGLER, F.E.; SENGSTAG, C. Metabolism of promutagens catalyzed by Drosophila melanogaster CYP6A2 enzyme in Saccharomyces cerevisiae. Environmental and Molecular Mutagenesis, v. 27, p. 46-58.

SANTOS, F. V.; TUBALDINI, F. R.; CÓLUS, I. M. S.; ANDRÉO, M. A.; BAUAB, T.M.; LEITE, C. Q. F.; VILEGAS, W.; VARANDA, E. A. Mutagenicity of Mouriri pusa Gardner and Mouriri elliptica Martius. Food and Chemical Toxicology, v. 46, p. 2721-2727, 2008.

SANTOS, J. H.; GRAF, U.; REGULY, M. L.; ANDRADE, H. H. R. The synergistic effects of vanillin on recombination predominate over its antimutagenic action in relation to MMC-induced lesions in somatic cells of Drosophila melanogaster. Mutation Research/Genetic Toxicology and Environmental Mutagenesis, v. 444, p. 355365, 1999.

STADLBAUER, V.; FICKERT, P.; LACKNER, C.; SCHMERLAIB, J.; KRISPER, P.; TRAUNER, M.; STAUBER, R. E. Hepatotoxicity of NONI juice: Report of two cases. World Journal of Gastroenterology, v. 11 (30), p. 4758-4760, 2005.

SU, B. N.; PAWLUS, A. D.; JUNG, H.; KELLER, W. J.; MCLAUGHLIN, J. L.; KINGHORN, A. D. Chemical Constituents of the Fruits of Morinda citrifolia (Noni) and their Antioxidant Activity. Journal of Natural Products, v. 68, p. 592-595, 2005. 
Franchi, L. P. et al./Revista Eletrônica de Farmácia Vol 5(3), 46 - 53, 2008.

VERSCHAEVE, L.; VAN STADEN, J. Mutagenic and antimutagenic properties of extracts from South African traditional medicinal plants Journal of Ethnopharmacology, v. 119, p. 575-587, 2008.

WANG, M. Y. \& SU, C. Cancer preventive effect of Morinda citrifolia (Noni). Annals of the New York Academy of Sciences, v. 952, p. 161-168, 2001.

WATERS, M. D.; BRADY, A. L.; STACK, H. F.; BROCKMAN, H. E. The concepty of activity profiles of mutagens. Antimutagenesis and Anticarcinogenesis Mechanism, v.52, p. 87-104, 1990.

WESTENDORF, J.; EFFENBERGER, K.; IZNAGUEN, H.; BASAR, S. Toxicological and Analytical Investigations of Noni (Morinda citrifolia) Fruit Juice. Journal of Agricultutal and Food Chemistry, v. 55, p. 529-537, 2007.

WÜRGLER, F. E.; SOBELS, F. H.; VOGEL, E. Drosophila as assay system for detecting genetic changes. Handbook of Mutagenicity Test Procedures, p. 335-373, 1984.

YÜCE, B.; GÜLBERG, V.; DIEBOLD, J.; GERBES, A.L. Hepatitis Induced by Noni Juice from Morinda citrifolia: A Rare Cause of Hepatotoxicity or the Tip of the Iceberg? Digestion, v. 73, p. 167-170, 2006. 